\section{IDENTIFICACIÓN DE LOS FACTORES SUBYACENTES DEL RIESGO DE DESASTRES EN EL NIVEL COMUNAL EN CHILE}

\author{
Natalia Silva Bustos ${ }^{1, *}$
}

\section{RESUMEN}

El articulo aborda el diseño e implementación de una herramienta que fue diseñada a través de un proceso multisectorial participativo, en el que, considerando criterio de expertos en sus temáticas y con conocimiento en gestión del riesgo de desastres, se jerarquizaron diversos factores que inciden en prácticas tendientes a la reducción del riesgo de desastres desde una perspectiva local e integral en el contexto de Chile. El objetivo del instrumento es apoyar la gestión municipal en la identificación, caracterización y abordaje de aquellas variables endógenas y exógenas presentes en el territorio comunal que condicionan eventuales escenarios de riesgo de desastres. Se consideran cuatro dimensiones para la categorización de los factores subyacentes del riesgo, las que corresponden a: gobernanza; ordenamiento territorial; condiciones socioeconómicas y demográficas; y recursos naturales y cambio climático. Dichas dimensiones agrupan un conjunto de cuarenta y un factores que se seleccionaron por su incidencia en la reducción del riesgo de desastres, los que se caracterizan a través de la aplicación de la encuesta y posterior procesamiento numérico de los resultados, mediante método multicriterio, generando un perfil único para cada municipio, orientando al equipo interdisciplinario a focalizar medidas estructurales y no estructurales en aquellos factores pesquisados tras la autoevaluación.

\section{PALABRAS CLAVE}

Impulsores de riesgo, Gobernanza local, Vulnerabilidad, Cambio climático, Planificación urbana

\section{IDENTIFYING THE UNDERLYING RISK FACTORS OF LOCAL COMMUNITIES IN CHILE}

\section{ABSTRACT}

The paper addresses the design and application of a tool that was built through a participatory multisectoral process in the context of Chile and from a local and integral perspective. The tool aims to support local governments in identifying, characterizing and approaching the endogenous and exogenous variables that condition disaster risks in their territories. The design process was based on experts' criteria and literature on disaster risk management, while various factors that affect disaster impacts and risk reduction practices were prioritized. Four dimensions were considered for the categorization of underlying risk factors: governance; territorial ordering; socio-economic and demographic conditions; and natural resources and climate change. These dimensions were sorted in forty-one variables that were selected due to their incidence in disaster risk reduction. Such variables were characterized through the application of survey to local governments and the subsequent numerical processing of the results through a multicriteria method. This generated a unique profile for each municipality that provide guiding principles to the interdisciplinary team to focus local interventions -structural and non-structural.

\section{KEYWORDS}

Risk drivers, Governance, Vulnerability, Climate change, Urban planning
1. Oficina Nacional de Emergencia del Ministerio del Interior, Santiago, Chile.

*Autor de correspondencia: nsilvab@onemi.gov.cl

\section{RECIBIDO}

17 de julio de 2019

\section{ACEPTADO}

14 de noviembre de 2019

\section{PUBLICADO}

1 de enero de 2020

\section{Formato cita}

Recomendada (APA):

Silva Bustos, N. (2020). Identificación de los Factores Subyacentes del Riesgo de Desastres en el Nivel Comunal en Chile. Revista de Estudios Latinoamericanos sobre Reducción del Riesgo de Desastres REDER, 4(1), pp.21-34.

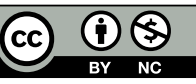

Todos los artículos publicados en REDER siguen una política de Acceso

Abierto y se respaldan en una Licencia CreativeCommons Atribución-NoComercial 4.0 Internacional.

\section{Revista de Estudios}

Latinoamericanos sobre Reducción del Riesgo de Desastres (REDER)

Diseño: Lupe Bezzina Tipografía: Hospital 


\section{CONTEXTO GENERAL}

Los desastres no son naturales, sino un producto de desarrollos que se enfrentan al riesgo teniendo un impacto profundo en el bienestar y los medios de vida de las comunidades locales. Además, si los desastres se entienden como la cristalización de las vulnerabilidades dentro de un grupo de individuos, determinada por factores subyacentes de riesgo $\neg$-como los atributos físicos del territorio y los cualitativos como la pobreza y la gobernanza (Dickson et al., 2012)- se abre una ventana para identificar y evaluar dichas vulnerabilidades y con ello, diseñar formas de reducirlas.

Como contexto físico general, Chile cumple con siete de los nueve criterios de vulnerabilidad al cambio climático según la Convención Marco de las Naciones Unidas sobre el Cambio Climático (CMNUCC, 2015). Además, dada su ubicación en el margen oriental del Cinturón de Fuego del Pacífico, Chile es uno de los países con mayor actividad volcánica y sísmica del mundo (Scholz, 2002). Asimismo, es uno de los países miembros de la OCDE más expuestos a amenzas de origen natural, con un $54 \%$ de su población y un $12,9 \%$ de su superficie total expuesta a tres o más tipos de amenazas (Dilley et al., 2005).

Las consecuencias de estas condiciones físicas se proyectan de diferentes maneras (Tironi, 2014) y se manifiestan en varios niveles, lo que puede obstaculizar el desarrollo sostenido del país. Por lo tanto, Chile se enfrenta constantemente al desafío de aprender de sus experiencias y fortalecer un enfoque de gestión del riesgo de desastres (GRD), con énfasis en el fortalecimiento de la resiliencia de las comunidades y sus gobiernos locales.

Así, en los últimos años, las políticas chilenas para la reducción del riesgo de desastres (RRD) han tenido un desarrollo activo y una alineación con las políticas globales, tales como: la Agenda 2030 para el Desarrollo Sostenible, el Acuerdo de París, el Marco de Sendai para la RRD 20152030 y su antecesor, el Marco de Acción de Hyogo 2005-2015, la Nueva Agenda Urbana - Hábitat III, entre otras. En consecuencia, Chile tiene una Política Nacional para la Gestión del Riesgo de Desastres y un Plan Estratégico Nacional que fue desarrollado por la Plataforma Nacional para la Reducción del Riesgo de Desastres, una entidad formada en Chile desde el año 2012 y que es el mecanismo más importante de coordinación intersectorial para la formulación, implementación y evaluación de la política pública en esta materia.

La Plataforma Nacional para la Reducción del Riesgo de Desastres es una piedra angular para los esfuerzos de RRD en Chile. En este sentido, se hizo cargo de una de las prioridades establecidas en la Política Nacional como es la reducción de los factores subyacentes del riesgo (FSR), desarrollando la Metodología de Identificación y Caracterización de los Factores Subyacentes de Riesgo a nivel local en el año 2016. Esta herramienta se aplica en el país a partir del año 2017 con la estrecha colaboración entre la Oficina Nacional de Emergencia del Ministerio del Interior y Seguridad Pública (ONEMI) y los gobiernos locales del país.

Por lo tanto, este artículo presenta los antecedentes y el contexto de la metodología, para describir luego sus componentes y resultados preliminares, lo que apunta a proporcionar un nuevo enfoque de evaluación de la vulnerabilidad de las comunidades locales para una mejor toma de decisiones para reducir el riesgo de desastres. La metodología se formuló revisando y considerando las metodologías aplicadas en diferentes regiones del mundo', que generalmente coinciden en las dimensiones que agrupan los principales factores subyacentes del riesgo de desastres. Sin embargo, el tratamiento numérico de las variables obedece a un procesamiento estadístico novedoso, que proporciona robustez al modelo y, por lo tanto, a los resultados obtenidos.

\section{ANTECEDENTES}

Como O'Keefe, Westgate y Wisner (1976) sugirieron que los desastres no son naturales, las comunidades son un elemento activo y responsable de administrar el riesgo (Twigg, 2009) como una construcción social (Wisner et al., 2004) y también que la vulnerabilidad individual, como un factor de riesgo relevante, se explica principalmente por las características sociales y un proceso de desarrollo inadecuado (Lavell, 2000) más que simplemente el entorno físico, es decir, el entorno físico no determina el desastre por sí mismo.

Bajo este enfoque holístico, la noción de los factores subyacentes de riesgo se refiere a la condición de vulnerabilidad inherente, es decir, aquellas condiciones que prevalecen en las comunidades que favorecen o facilitan sus efectos (Briguglio, 2003).

El Informe de Evaluación Global sobre la Reducción del Riesgo de Desastres (GAR, por sus siglas en inglés, 2009) se refiere a los factores subyacentes de riesgos o impulsores de riesgo

1. La metodología más similar a esta propuesta es la que calcula el índice de reducción de riesgo en África Occidental. Sin embargo, la mayoría de las metodologías encontradas en la revisión bibliográfica corresponden a un análisis cualitativo y/u obtenido de la percepción frente a una lista de condiciones y capacidades de afrontamiento relacionadas con el riesgo a nivel local. Además, la novedad de este método es que no se enfoca en un riesgo/exposición particular ni está estructurado en torno a las fases de la gestión del riesgo, sino que presenta una vista integral de todas las dimensiones básicas que determinan una posible situación de riesgo. 
como "procesos, tanto físicos como sociales, que contribuyen, conducen o determinan de manera importante la construcción, creación o existencia de condiciones. del riesgo de desastres en la sociedad".

El Marco de Sendai identificó las brechas relacionadas con los FSR que indican que "las acciones más dedicadas deben centrarse en abordar los factores subyacentes del riesgo de desastres, como las consecuencias de la pobreza y la desigualdad, el cambio y la variabilidad climática, la urbanización rápida y no planificada, la gestión deficiente de la tierra y la composición de factores como los cambios demográficos, débiles arreglos institucionales, políticas no informadas sobre riesgos, falta de regulación e incentivos para la inversión privada para la reducción del riesgo de desastres, cadenas de suministro complejas, disponibilidad limitada de tecnología, usos no sostenibles de los recursos naturales, ecosistemas en declive, pandemias y epidemias".

Además, la Agenda 2030 para el Desarrollo Sostenible, enfatiza la importancia de que su implementación debe alinearse con los otros compromisos globales afines. Esta relación indivisible entre los objetivos perseguidos por cada uno de ellos es crucial para lograr el desarrollo integral de los países, considerando las dimensiones sociales, económicas y ambientales; reducir la vulnerabilidad y mejorar la capacidad de recuperación (CMNUCC, 2017).

\section{METODOLOGÍA}

\section{Diseño del Instrumento}

La definición del propósito y diseño de esta herramienta fue el resultado de un año de trabajo realizado por un grupo de expertos nacionales de la sociedad civil organizada, sector público, privado y académico miembros de la Plataforma Nacional para la Reducción del Riesgo de Desastres. En su etapa inicial trató de capturar las diferentes visiones sectoriales con un enfoque multidimensional que considera las diferencias territoriales, advirtiendo que los gobiernos locales son actores claves para lograr la reducción del riesgo y también son los primeros responsables de administrar su territorio y comunidad.

Para ayudar a los gobiernos locales a comprender su situación, el grupo de expertos estableció que la evaluación debe consistir en un autodiagnóstico guiado mediante la aplicación de una encuesta.

El propósito del instrumento es favorecer y fortalecer, de manera oportuna y relevante, los procesos de diseño, planificación, inversión, ejecución y evaluación de diversas iniciativas públicas y privadas en el territorio, fortaleciendo así las capacidades preventivas, de respuesta y adaptación de las comunidades, instituciones y territorio. El instrumento permite la generación de un diagnóstico comunitario único; la definición de una línea de base que lleva a la evaluación del progreso en diferentes niveles; y la proporción de recomendaciones específicas de gestión de riesgo a los municipios.

El instrumento completo incluye un enfoque inicial para el personal del gobierno local, un documento guía (fuentes de información sugeridas y terminología oficial) y cuatro folletos con las preguntas, correspondientes a cada una de las dimensiones encuestadas.

Las dimensiones consideradas son: gobernanza, planificación territorial, condiciones socioeconómicas y demográficas, y cambio climático y recursos naturales. Las cuatro abarcan 41 variables que se consideran relevantes para la disciplina RRD, como se describe en las tablas a continuación.

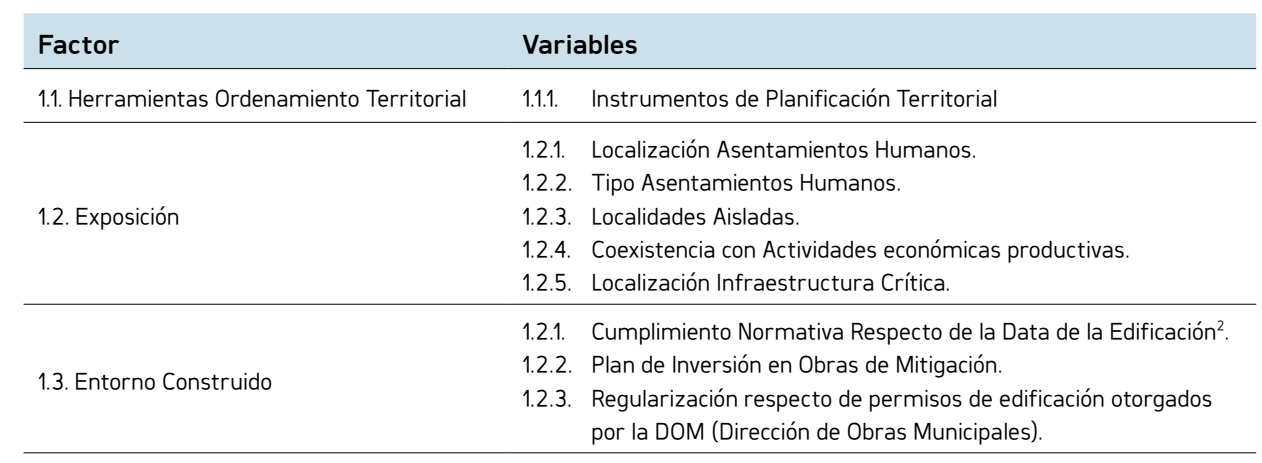

Tabla l. Dimensión de Ordenamiento Territorial

Fuente: Autora, 2020 


\begin{tabular}{|c|c|c|}
\hline Factor & \multicolumn{2}{|c|}{ Variables } \\
\hline 2.1. Variabilidad Climática & $\begin{array}{l}\text { 2.1.1. } \\
\text { 2.1.2. }\end{array}$ & $\begin{array}{l}\text { Patrón de Comportamiento de los Eventos Extremos del Clima. } \\
\text { Actualización y Acceso a Información sobre impacto del cambio } \\
\text { climático. }\end{array}$ \\
\hline 2.2. Degradación Ambiental & $\begin{array}{l}1.2 .1 \\
1.2 .2 \\
1.2 .3 \\
1.2 .4 \\
1.2 .5\end{array}$ & $\begin{array}{l}\text { Localización Asentamientos Humanos. } \\
\text { Tipo Asentamientos Humanos. } \\
\text { Localidades Aisladas. } \\
\text { Coexistencia con Actividades económicas productivas. } \\
\text { Localización Infraestructura Crítica. }\end{array}$ \\
\hline
\end{tabular}

Tabla 2. Dimensión Cambio Climático y Recursos Naturales

Fuente: Autora, 2020

\begin{tabular}{|c|c|}
\hline Factor & Variables \\
\hline 3.1. Pobreza e Inequidad & $\begin{array}{l}\text { 3.1.1. Incidencia de la Pobreza Multidimensional }{ }^{3} \text {. } \\
\text { 3.1.2. Incidencia de la Pobreza por Ingresos }{ }^{4} \text {. } \\
\text { 3.1.3. Calificación Socioeconómica. }\end{array}$ \\
\hline 3.2. Propiedades Demográficas & $\begin{array}{l}\text { 3.1.1. Índice de Dependencia Demográfica }{ }^{5} \text {. } \\
\text { 3.1.2. Población en Situación de Discapacidad. } \\
\text { 3.1.3. Población en Situación de Calle. } \\
\text { 3.1.4. Población Inmigrante Internacional. }\end{array}$ \\
\hline
\end{tabular}

Tabla 3: Dimensión de Condiciones Socioeconómicas y demográficas Fuente: Autora, 2020

\begin{tabular}{|c|c|c|c|}
\hline \multicolumn{2}{|c|}{ Factor } & \multicolumn{2}{|c|}{ Variables } \\
\hline \multirow{13}{*}{4.1.} & \multirow{13}{*}{ Institucionalidad } & 4.1.1. & Enfoque Inclusivo en ámbitos de la Gestión Municipal. \\
\hline & & 4.1.2. & Gestión Local y Adaptación al Cambio Climático. \\
\hline & & 4.1.3. & Carácter de la Participación Ciudadana. \\
\hline & & 4.1.4. & Capacidades Municipales para la RRD. \\
\hline & & & Sub-variables: \\
\hline & & & 4.1.4.1. Estructura Municipal. \\
\hline & & & 4.1.4.2. Capacitación Comunal. \\
\hline & & & 4.1.4.3. Autonomía Financiera y en la Toma de Decisiones. \\
\hline & & & 4.1.4.4. Instrumentos Locales. \\
\hline & & & 4.1.4.5. Comité de Protección Civil. \\
\hline & & 4.1.5. & $\begin{array}{l}\text { Seguridad Pública y Protección a las Personas en Situaciones de } \\
\text { Emergencias. }\end{array}$ \\
\hline & & 4.1.6. & Mecanismos de Rendición de Cuentas. \\
\hline & & 4.1.7. & Cobertura de Programas Sociales. \\
\hline \multirow{4}{*}{4.2.} & \multirow{4}{*}{ Cohesión Social } & 4.1.1. & Construcción de Información Oficial. \\
\hline & & 4.1.2. & Representación Ciudadana. \\
\hline & & 4.1.3. & Pertenencia Social. \\
\hline & & 4.1.4. & Organizaciones de la Sociedad Civil. \\
\hline \multirow[b]{2}{*}{4.3.} & \multirow[b]{2}{*}{ Compromiso Sectorial } & 4.1.1. & Responsabilidad en la Inversión Privada. \\
\hline & & 4.1.2. & $\begin{array}{l}\text { Mecanismos de Transferencia del Riesgo (Protección } \\
\text { Financiera). }\end{array}$ \\
\hline
\end{tabular}

Tabla 4. Dimensión de Gobernanza

Fuente: Autora, 2020

\section{Construcción del Modelo}

La construcción del modelo se realizó utilizando un método de procesamiento estadístico multicriterio denominado Proceso Jerárquico Analítico (AHP, Analytical Hierarchical Process) aplicado a las variables en todos los niveles mencionados anteriormente. La importancia relativa, es decir, las ponderaciones específicas de cada componente y las respuestas en el modelo se asignaron por criterios de expertos teniendo en cuenta su incidencia en la gestión local del riesgo de desastres. Una de las posibilidades que contribuyen las metodologías de criterios múltiples es la diversidad de factores que pueden integrarse en el proceso de evaluación. Su particularidad es la forma de transformar medidas y percepciones en una sola escala para comparar los elementos y establecer órdenes de prioridad que permiten agregar los efectos de un proyecto en una métrica común (Contreras et al., 2007).
3. Compuesto por cinco dimensiones relevantes de bienestar: (1) Educación (2) Salud; (3) Trabajo y Seguridad Social; (4) Vivienda y Medio Ambiente; y, (5) Redes y Cohesión Social. Los hogares que acumulan $22.5 \%$ o más de las privaciones se encuentran en una situación de pobreza multidimensional. Las personas consideradas pobres son aquellas que forman parte de los hogares cuyo ingreso mensual total es menor que la "línea de pobreza por persona equivalente", o el ingreso mínimo establecido para satisfacer las necesidades básicas de alimentos y no alimentos en el mismo período, según el número de miembros del dueño de casa.

4. Las personas consideradas pobres son aquellas que forman parte de los hogares cuyo ingreso mensual total es menor que la "línea de pobreza por persona equivalente", o el ingreso mínimo establecido para satisfacer las necesidades básicas de alimentos y no alimentos en el mismo período, según el número de miembros del dueño de casa.

5. Se refiere a la relación entre el número de personas en edades dependientes (o a 14 años y mayores de 60 años) con la población económicamente activa (15 a 59 años) del municipio. 
Los cien vectores de prioridad, calculados entre cada par de componentes del modelo en cada nivel jerárquico realizado utilizando los pesos de la escala absoluta conocida como la Escala Fundamental de Saaty, permiten obtener la regla de medición (Figura 2). Se necesitaron seis comparaciones para cubrir las cuatro dimensiones, 45 comparaciones para los diez factores, 630 comparaciones para las 36 variables y 10 para las cinco sub-variables del modelo. El mismo ejercicio se llevó a cabo en cada rango de respuestas posibles: nulo, bajo, medio y alto; donde medio y alto corresponden al peor desempeño. La encuesta ofrece 4 respuestas posibles para cada una de las 41 variables solicitadas, que describen diferentes escenarios posibles (formulados por el mismo grupo de expertos, con la intención de capturar escenarios lo suficientemente genéricos para ser representativos en todo el territorio nacional), invitando al equipo municipal a identificarse con solo uno de ellos.

Como resultado del proceso iterativo de ponderaciones, el grupo de expertos llegó a la conclusión de que el mayor peso estaba asociado a la dimensión de Gobernanza, ya que sienta las bases para todas las interrelaciones entre las partes interesadas y, por lo tanto, establece la fuerza potencial de las políticas públicas de RRD. El segundo peso más alto se asignó a la dimensión de Ordenamiento territorial, seguido por la dimensión de Condiciones socioeconómicas y demográficas, y por último la dimensión de Cambio climático y Recursos naturales, entendida como un modulador de amenazas y condiciones exógenas. La Figura 1 muestra los pesos de cada una de las dimensiones en el modelo genérico.

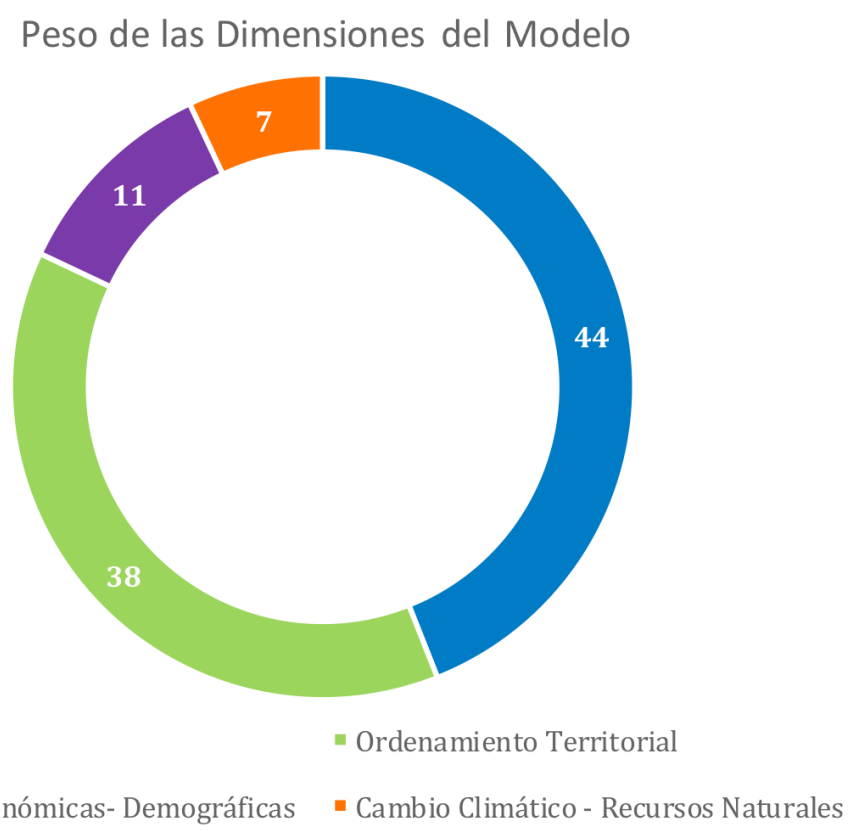

- Gobernanza

- Condiciones Socioeconómicas- Demográficas - Cambio Climático - Recursos Naturales

Figura 1. Pesos de las Dimensiones del Modelo Fuente: Autora, 2020

La jerarquía, base del método de multicriterio AHP, es una abstracción de la estructura de un sistema de decisión, que permite estudiar las interacciones funcionales de sus componentes y el impacto global en un sistema. Este método genera indicadores para cada dimensión y un indicador general para cada gobierno local encuestado. El indicador global se denominó Índice Comunal de Factores Subyacentes de Riesgo (ICFSR).

Cada gobierno local encuestado obtiene una clasificación basada en el ICFSR obtenido. Las estimaciones de las categorías son: niveles de riesgo mínimo, bajo, medio y alto, obtenidos después de un proceso numérico de definición de umbrales teniendo en cuenta el criterio de los expertos.

Después de la construcción del modelo jerárquico, el equipo de ONEMI aplicó la encuesta en 4 municipios considerados como de control, esto, dado el conocimiento que las oficinas regionales de ONEMI (como coordinador del Sistema Nacional de Protección Civil de Chile) tiene de los municipios. En este sentido, una vez que se obtuvieron los resultados, se hicieron algunos ajustes en el peso de 
algunas variables, de modo que el modelo replicó el comportamiento esperado en esas comunas bien conocidas en términos de RRD (dos municipios en la Región Metropolitana y dos en el sur del país, contextos rurales y urbanos y con diferentes tamaños de población y capacidades disponibles). Una vez que se realizó esta calibración, se decidió iniciar la aplicación masiva y gradual del instrumento, que, por cierto, siempre que los resultados nacionales de la encuesta estén disponibles, se puede revisar, actualizar y mejorar si se considera necesario para siguientes aplicaciones que permitan comparaciones temporales en cuanto a gestión en la comuna.

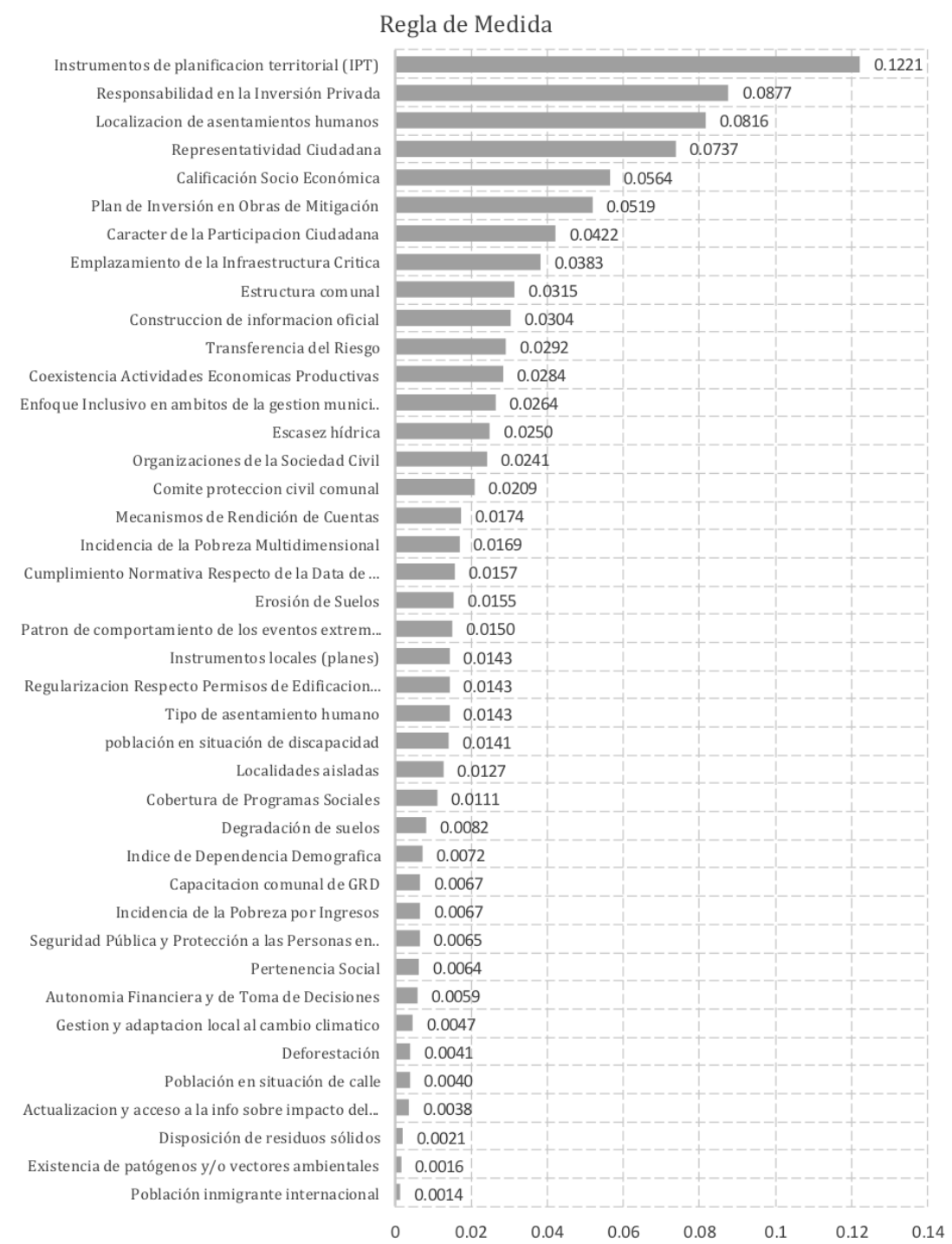

Figura 2. Regla de Medida construida mediante criterio experto, usando método AHP Fuente: Autora, 2020

\section{Aplicación de la Encuesta}

Antes de la aplicación de la encuesta, ONEMI contacta a su punto focal en el gobierno local para coordinar el taller (modalidad de aplicación de la encuesta) y para proporcionar, por adelantado, todos los materiales en formatos electrónicos, incluidas las fuentes de información oficial. La aplicación requiere que un equipo de gobierno local esté compuesto al menos por los siguientes funcionarios: obras civiles, desarrollo social, protección civil, operaciones de emergencia, medio ambiente, planificación, participación comunitaria y programas enfocados en grupos prioritarios, entre otros funcionarios municipales según los diferentes contextos y capacidades disponibles.

Por lo general, la aplicación de la encuesta no excede las dos horas en compañía de ONEMI, que actúa como facilitador y brinda guía y apoyo a los equipos locales para completar el cuestionario utilizando las fuentes de información oficiales, lo que lleva a criterios uniformes y respuestas confiables. 
Cada folleto debe ser firmado por aquellos que respondieron cada dimensión, y todas las preguntas deben ser respondidas para proporcionar los resultados, informes y recomendaciones contempladas por el instrumento.

\section{Procesamiento}

ONEMI realiza un proceso de control y garantía de calidad que contrasta cierta información oficial con las respuestas proporcionadas antes de continuar con el análisis numérico de la encuesta.

El propósito del procesamiento estadístico es proporcionar a los gobiernos locales un Informe Individual, que incluye los siguientes elementos:

a. Índice Comunal de Factores Subyacentes de Riesgo (ICFSR) que indica el rango en el que se clasifica, de la manera en que se ejemplifica en la Figura 3.

b. Gráfico de rendimiento por dimensión, que permite conocer el nivel en el que se ubica el municipio para cada una de las cuatro dimensiones (línea azul), como se ilustra en la Figura 4.

c. Perfil de desempeño por variable, que muestra el comportamiento del municipio para cada una de las 41 variables medidas. En rojo se destacan las variables de alta incidencia, que corresponde a las variables mal evaluadas y también cuyo peso es relevante en el modelo, como se muestra en la Figura 5. De esta manera, el Municipio recibe asesoramiento sobre la priorización de dichas variables, apelando al uso eficiente de los recursos, tanto en medidas estructurales como no estructurales.

d. Conjunto de recomendaciones genéricas para aquellas variables con una alta incidencia para incorporarlas como parte de los objetivos de gestión local. Idealmente, se espera que en un período de no más de 3 años se vuelva a aplicar la encuesta, por lo tanto, es importante que el equipo municipal reciba apoyo y asesoramiento técnico para alentar la consideración de las recomendaciones brindadas.

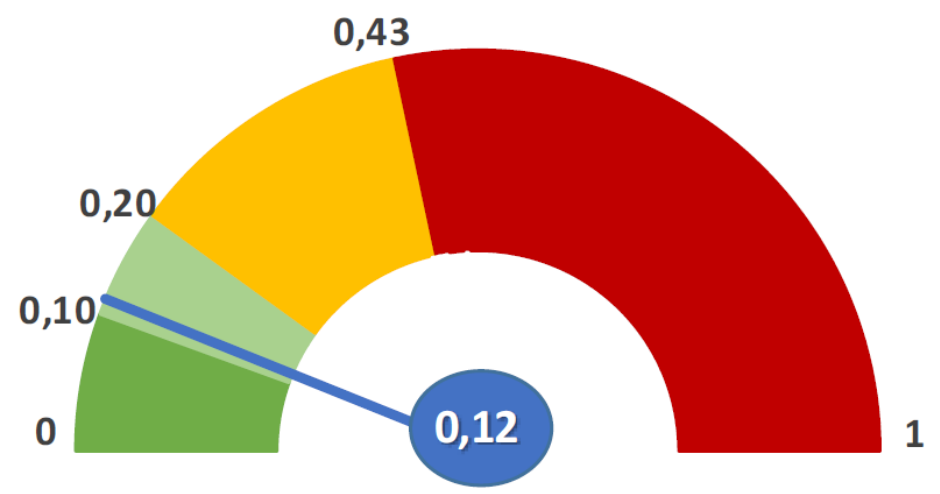
Nivel Mínimo:
ICFSR $\leq 10 \%(\leq 0.10)$
Nivel Bajo:
ICFSR $>10 \% \leq$ a $20 \%(0.11$ a 0.20$)$
Nivel Medio:
ICFSR $>20 \%<43 \%(0.21$ a 0.42$)$
Nivel Alto:
ICFSR $\geq 43 \%(\geq 0.43)$

Figura 3. Índice Comunal de Factores Subyacentes de Riesgo. Ejemplo de Municipio ICFSR $=0.12(12 \%)$, nivel de bajo riesgo Fuente: Autora, 2020 
Resultados por Dimensión, Municipio X

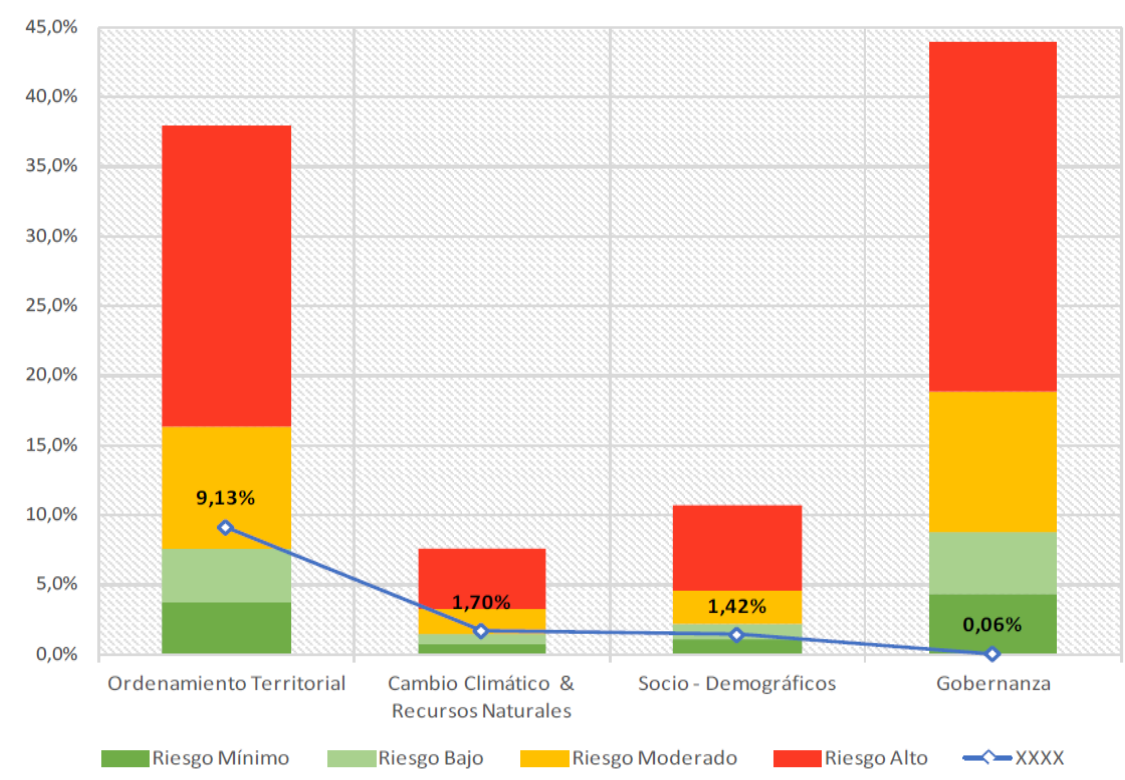

Figura 4. Resultados por Dimensiones. Ejemplo de municipio ICFSR = $0.12(12 \%)$, nivel de bajo riesgo. Fuente: Autora, 2020

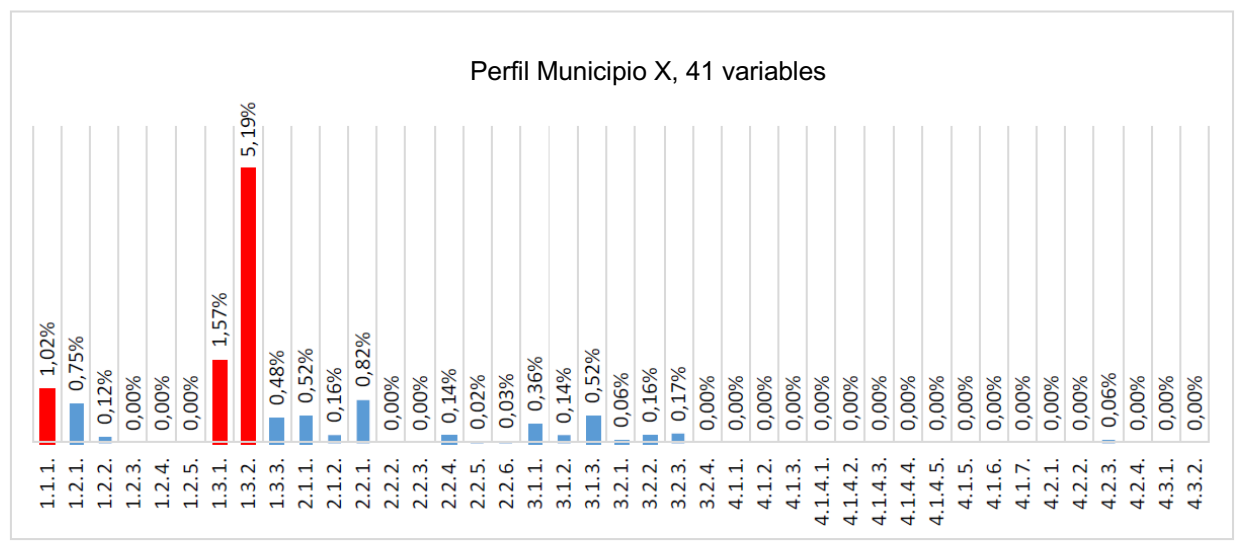

Variables de Alta Incidencia (pobremente evaluadas y de alto peso en el modelo)

Figura 5. Perfil por Variables. Ejemplo de municipio ICFSR = $0.12(12 \%)$, nivel de bajo riesgo Fuente: Autora, 2020

\section{RESULTADOS PARCIALES}

Después del proceso piloto y la calibración del instrumento, la aplicación de la encuesta se inició en el año 2017. Ese año se muestrearon un total de 60 municipios, distribuidos en todo el país de manera representativa (uno por cada provincia de cada región del país, cubriendo la división política administrativa de Chile continental). Los resultados, con respecto al ICFSR se muestran desde la Figura 6 a la Figura 10. Se espera que la cobertura completa de los 345 municipios de Chile se logre para fines del año 2019. 


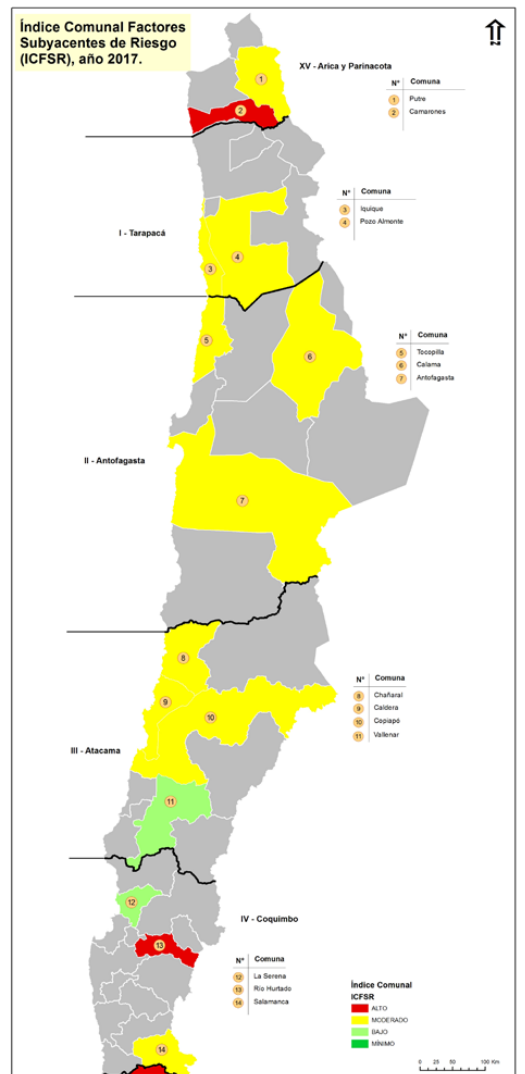

ICFSR $\leq 0.10$

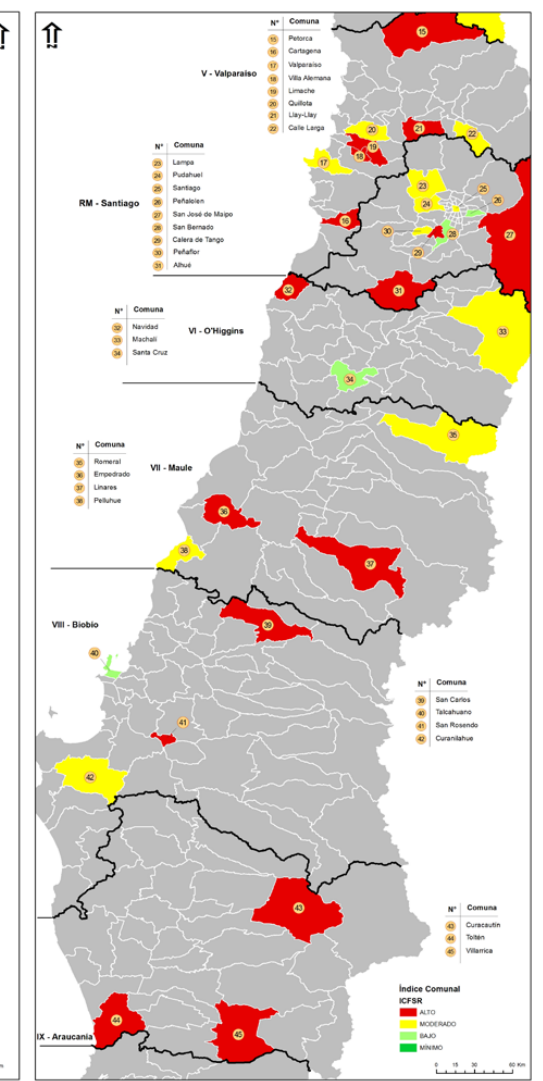

$0,21<$ ICFSR $<0.42$

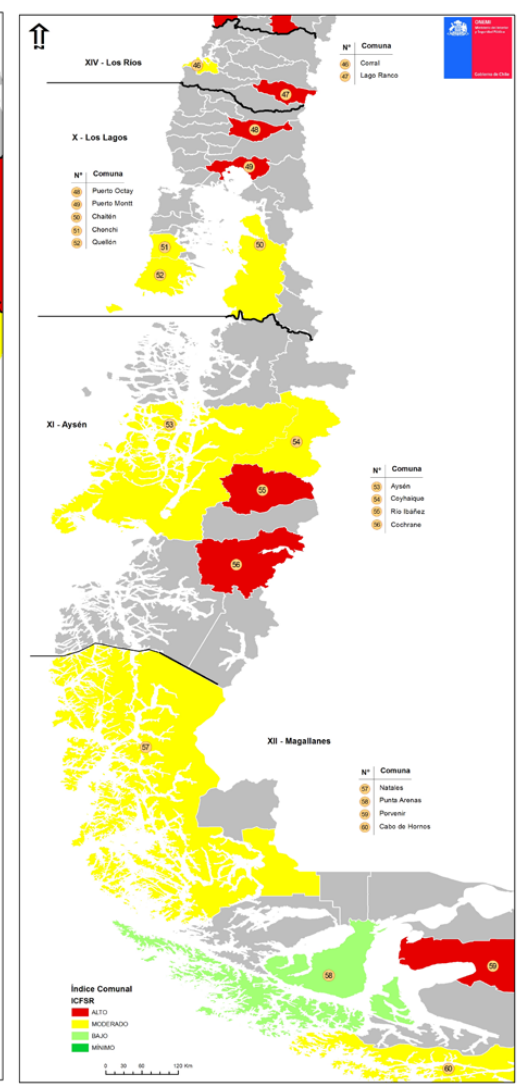

ICFSR $\geq 0.43$

Figura 6. Îndice Comunal de Factores Subyacentes de Riesgo (ICFSR), muestra 2017 (60 municipios). Índice agregado Fuente: Autora, 2020

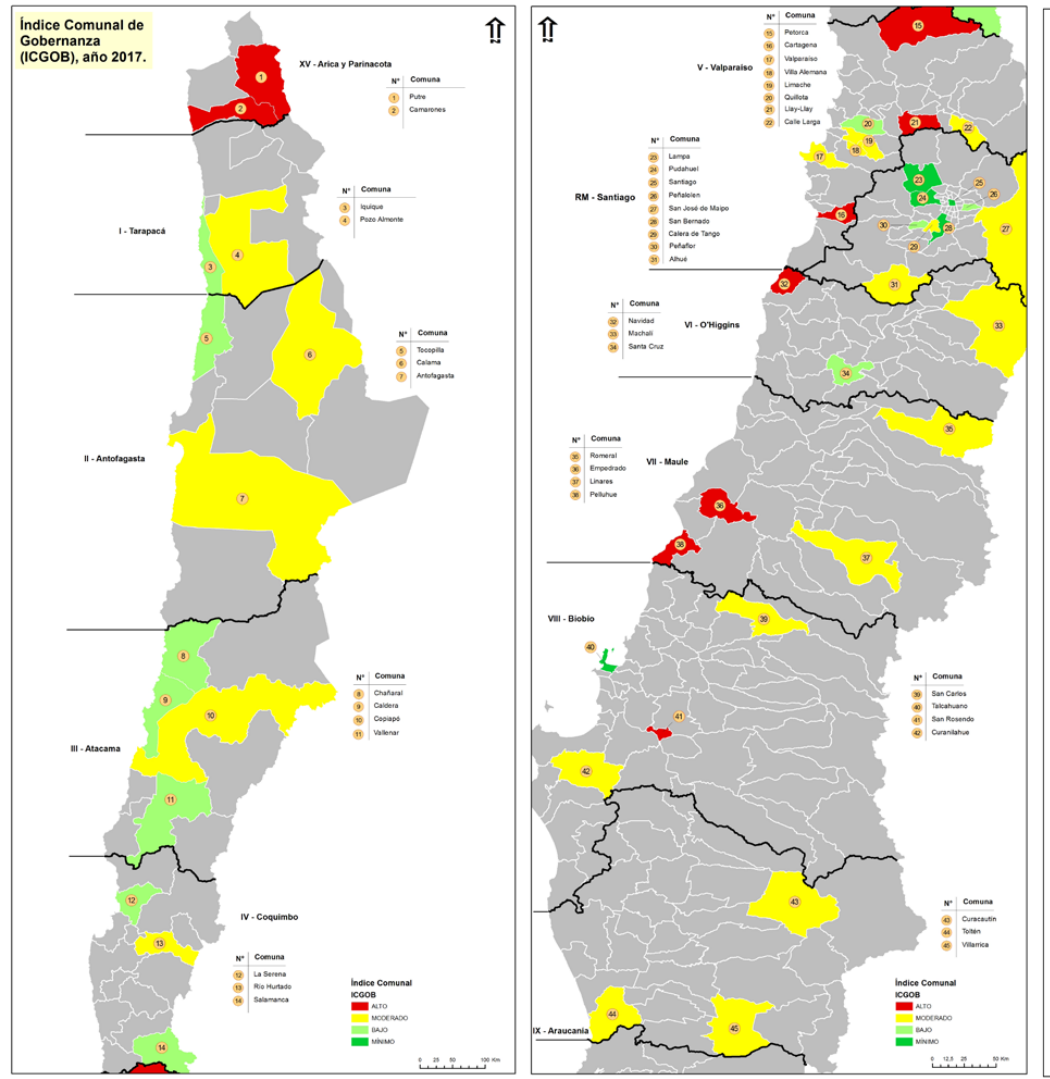

IC_GOB $\leq 4.39$

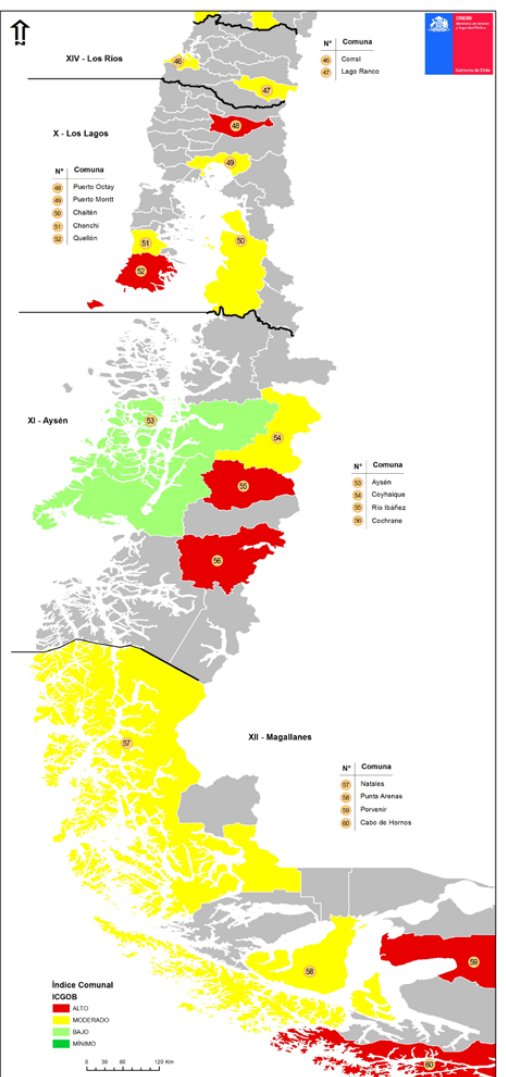

IC_GOB $\geq 1$

Figura 7. Índice Comunal de Factores Subyacentes de Riesgo (ICFSR), muestra 2017. Dimensión Gobernanza (ICGOB) 

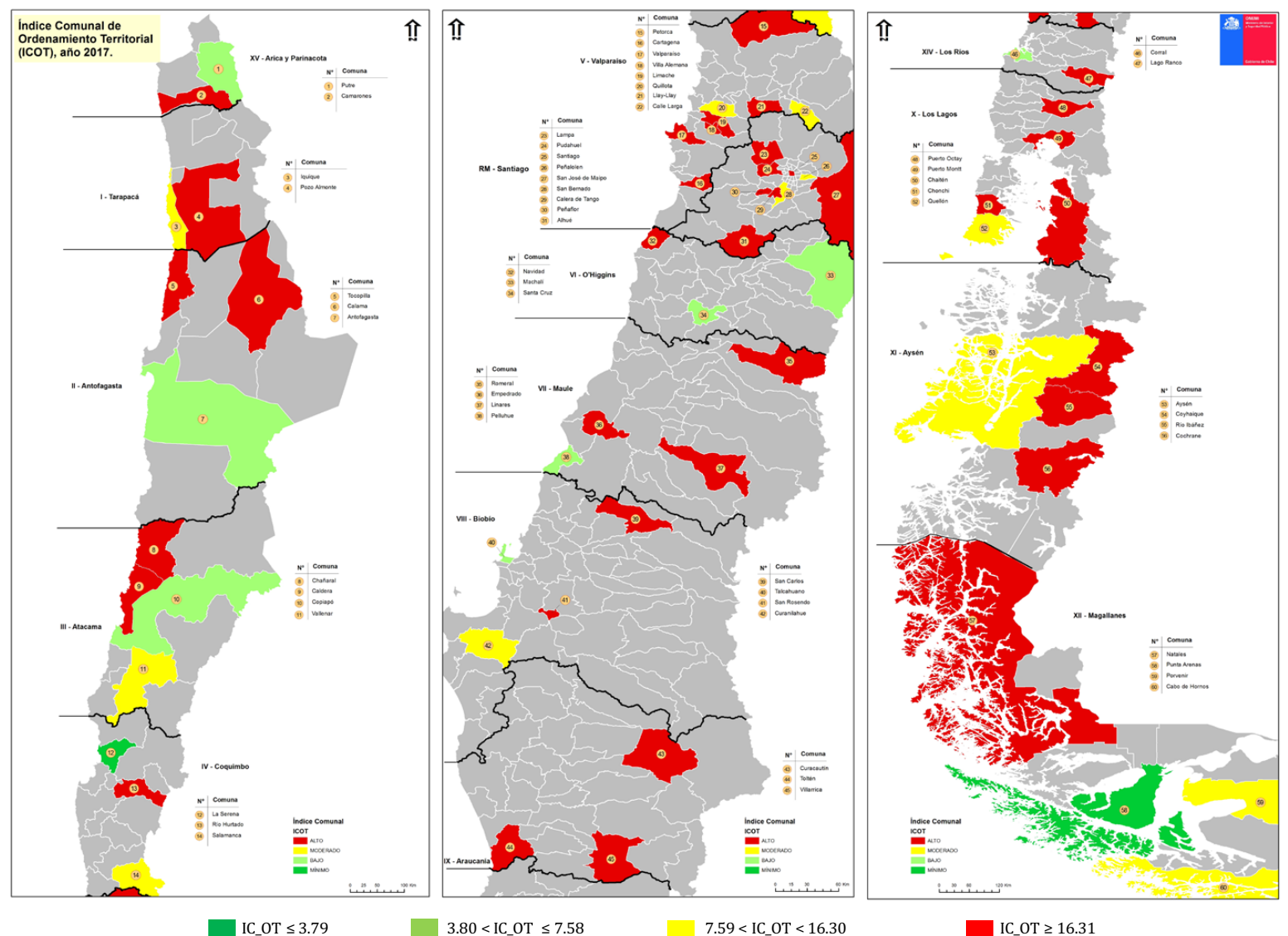

Figura 8. Índice Comunal de Factores Subyacentes de Riesgo (ICFSR), muestra 2017. Dimensión Ordenamiento Territorial (ICOT) Fuente: Autora, 2020

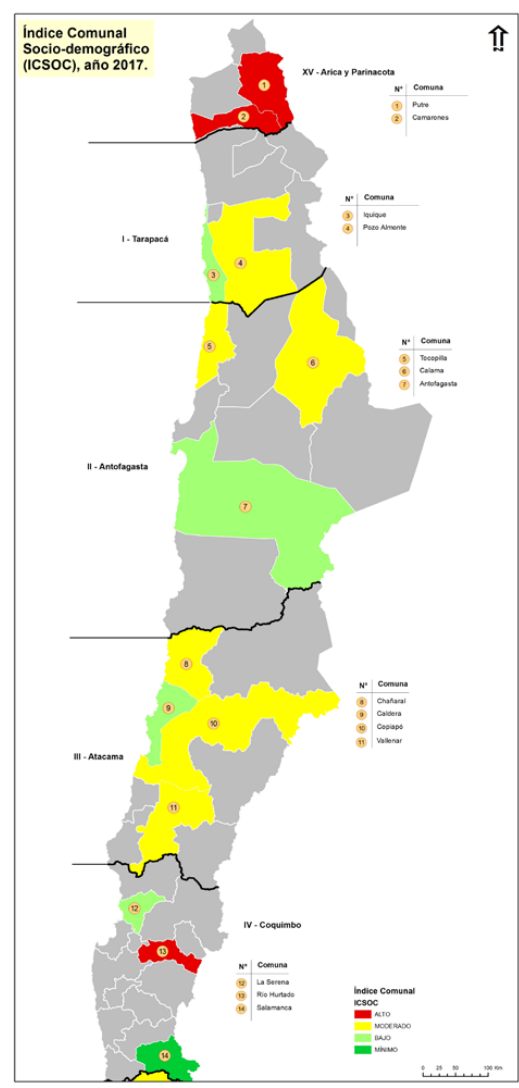

IC_SOC $\leq 1.07$

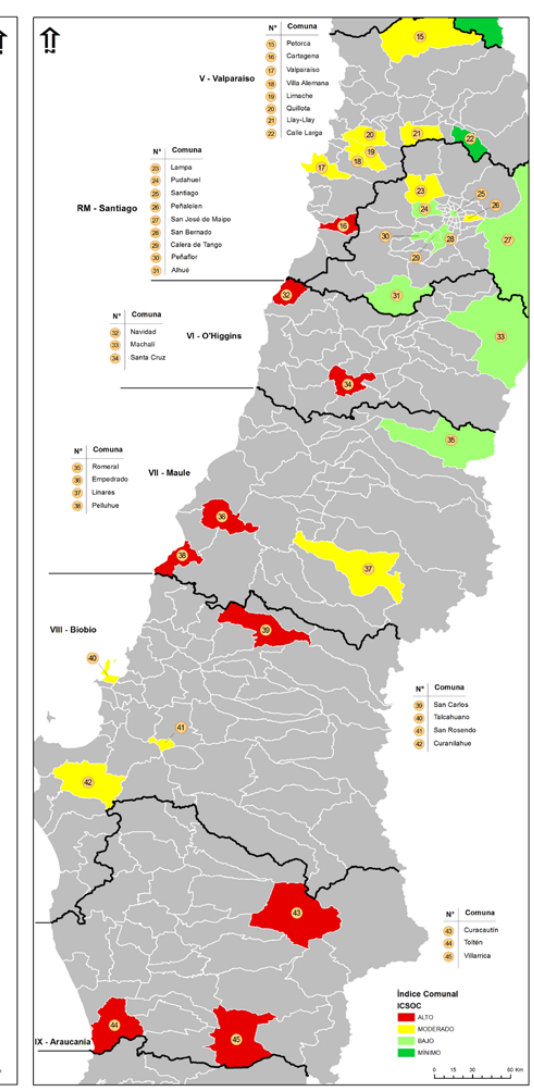

$1.08<$ IC_SOC $\leq 2.12$
$2.13<$ IC_SOC $<4.58$

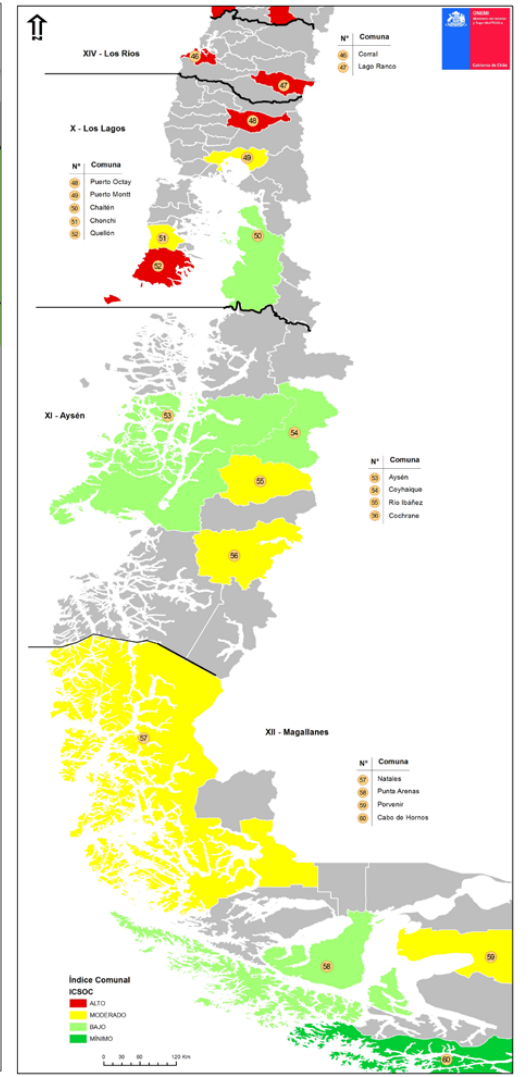

IC_SOC $\geq 4.59$ 


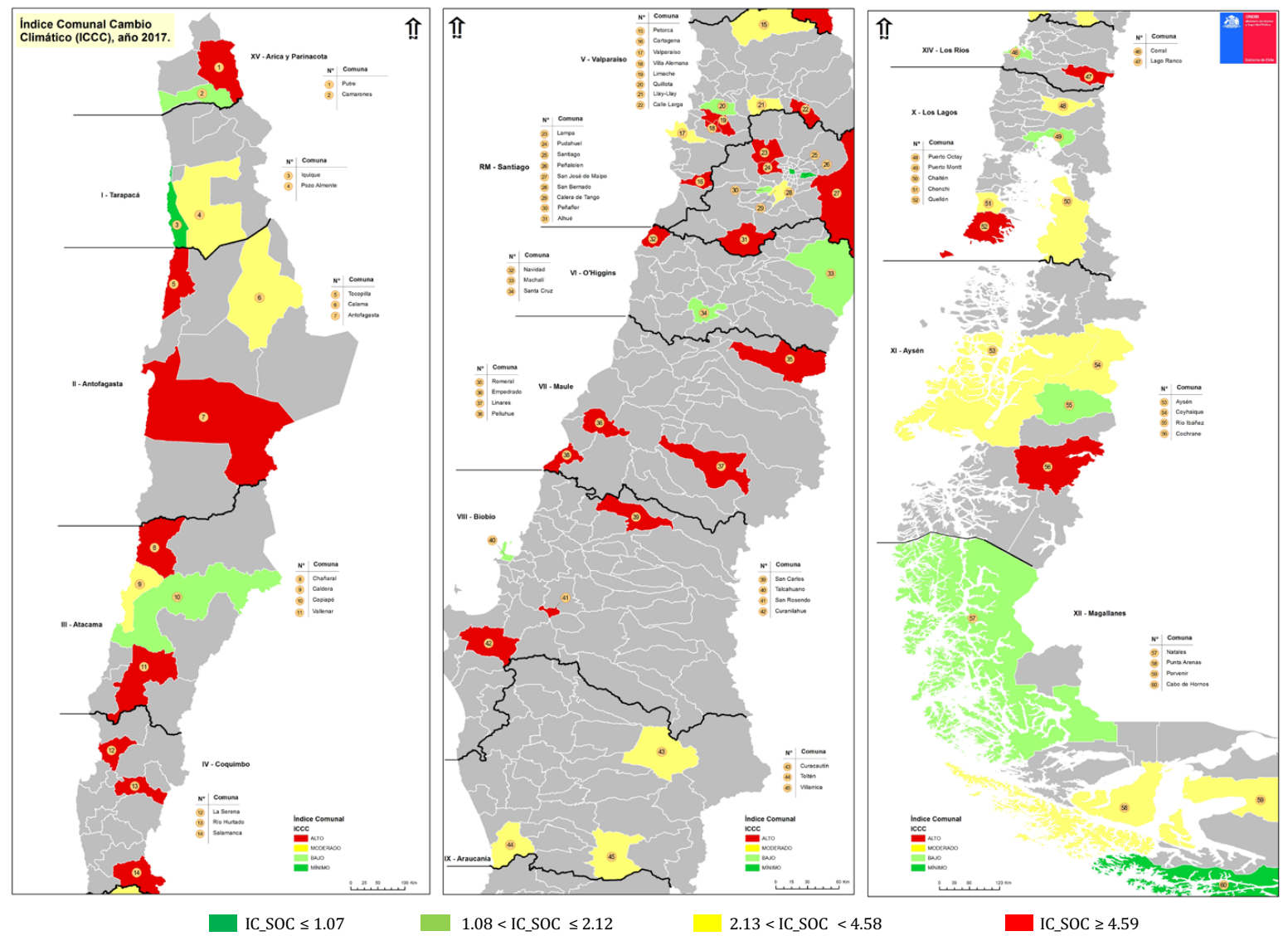

Figura 10. Índice Comunal de Factores Subyacentes de Riesgo (ICFSR), muestra 2017. Dimensión Recursos Naturales y Cambio Climático (ICCC) Fuente: Autora, 2020

La siguiente Figura 11 resume el desempeño de la gestión del riesgo obtenido por la muestra del año 2017. Los 60 municipios están clasificados por el ICFSR (valores de la Figura 6) de mejor a peor desempeño, de izquierda a derecha.

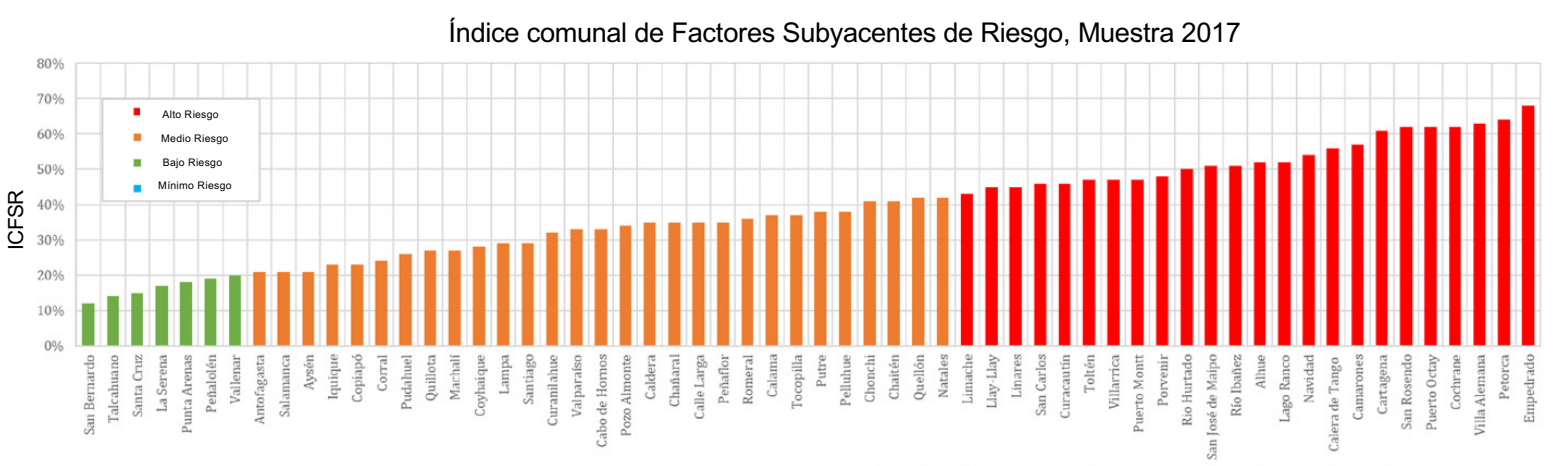

Figura 11. Índice Comunal de Factores Subyacentes de Riesgo (ICFSR) muestra 2017 Fuente: Autora, 2020

Esta figura muestra que ningún municipio obtuvo un nivel mínimo (ICFSR $\leq 10 \%$ o 0.10 ), aproximadamente el $10 \%$ de la muestra tiene un ICFSR bajo, el $50 \%$ de la muestra tiene un nivel moderado y el $40 \%$ mostró riesgo de alto nivel.

Es importante tener en cuenta que un mismo índice global (ICFSR de las dimensiones agregadas) se puede obtener de diferentes comportamientos (ejemplo municipios de Salamanca, Antofagasta y Aysén, los tres con igual índice 0.21 pero con realidades y contextos diferentes que se visualizan en sus respectivos reportes), por lo que se debe realizar una evaluación exhaustiva utilizando el perfil de desempeño para cada una de las 41 variables que permiten comprender las singularidades de cada gobierno local, dado que este perfil rescata las particularidades del territorio y actores que inciden en cada municipio. 
La muestra del 2017 corresponde al 20\% del total de municipios del país, involucra al 25\% de la población chilena, cubre todas las provincias del país y es en su mayoría de carácter urbano o semiurbano. Alrededor del $60 \%$ de los municipios seleccionados tienen un índice de pobreza de ingresos superior al porcentaje promedio nacional.

Respecto a los instrumentos de planificación urbana, el 73\% de la muestra indica tener un Plan Regulador Comunal, PRC (no necesariamente actualizado) y, respecto a las Estrategias de Desarrollo, el $95 \%$ de los municipios evaluados declara tener su Plan de Desarrollo Comunitario actualizado (PLADECO).

El 50\% de los municipios de la muestra tiene un alto nivel de dependencia del Fondo Común Municipal (es decir, baja generación de recursos propios y dependientes directos de la distribución del fondo), mientras que solo el $17 \%$ de los municipios de la muestra tiene una baja dependencia (alta capacidad para generar recursos propios).

Es importante enfatizar que lo que esta metodología busca es enfocarse en aquellas variables que tienen un alto impacto en el modelo. La razón de esto último es que una variable mal evaluada que pesa poco, su remediación puede no tener un impacto estratégico o práctico en la gestión local, como sí pudiera tener la atención de una variable mal evaluada, pero de alto peso en el modelo.

Los principales hallazgos después de analizar la muestra son:

a. Los municipios rurales a semiurbanos clasificados como de desarrollo medio o bajo, según el estándar nacional, presentan un nivel de alto riesgo en la dimensión de Ordenamiento Territorial.

b. Los municipios que muestran una dependencia moderada a baja del fondo municipal común tienen un riesgo bajo en todas las dimensiones evaluadas, en relación con una mayor autonomía financiera y la autogestión propia o de los recursos.

c. Los municipios con menores porcentajes de pobreza por ingreso se evalúan mejor en todas las dimensiones, mostrando un nivel bajo del ICFSR.

d. Un alto porcentaje de comunas con un bajo nivel del ICFSR tienen un bajo crecimiento urbano en los últimos 15 años.

\section{CONCLUSIONES}

El desarrollo de este instrumento representa un esfuerzo pionero para capturar las diferentes características que configuran la vulnerabilidad y guían la gestión eficaz del riesgo de desastres a nivel local. Abogamos porque la creación de esta herramienta permita a los gobiernos locales participar en el proceso de identificación, gestión y monitoreo de la situación de diagnóstico detectada después de la aplicación de la encuesta.

El instrumento representa beneficios directos según lo expuesto, pero también abarca numerosos beneficios indirectos como se observó durante su aplicación. Entre los últimos está el trabajo de interacción entre variadas áreas/departamentos de la estructura del municipio, la invitación a discutir y reflexionar de temas que no estén directamente asociados con la RRD, proporcionar antecedentes, cifras y datos actualizados y oficiales de cada municipio, solo para nombrar algunas bondades detectadas.

Su objetivo es fortalecer la gobernanza de los administradores locales para reducir el riesgo de desastres que presentan los factores detectados, permitiendo abordar las condiciones de vulnerabilidad existentes para aceptar, reducir, mitigar o transferir el riesgo local. Dado que existen amenazas y otras condiciones de base que van más allá de los límites administrativos, es crucial promover el trabajo colaborativo con otros gobiernos locales que comparten realidades similares, pudiendo promover proyectos o iniciativas e inversiones conjuntas, compartir buenas prácticas, involucrar y sensibilizar a actores externos al territorio comunitario.

Con base en los datos disponibles y las relaciones evidenciadas en diferentes países del mundo, Chile llevó a cabo el ejercicio de apropiación del concepto y adaptación al contexto nacional y cultural, construyendo una metodología estándar que puede ser adaptada y adoptada por otros países para identificar y caracterizar estas condiciones de manejo del riesgo, como son los factores subyacentes de riesgo. 
Dada la importancia del proceso de captura de información, también su complejidad potencial, ya que es un nuevo instrumento de gestión en el país y que requiere la participación transversal de funcionarios municipales, es que la encuesta consideró un proceso de acompañamiento realizado por ONEMI. Además, se espera que se haga un seguimiento de las recomendaciones entregadas en un plazo no mayor a tres años, comprendiendo el dinamismo de algunas condiciones de riesgo y los nuevos antecedentes que pueden surgir de las fuentes oficiales utilizadas en la encuesta. Por lo tanto, la importancia de acompañar a nivel nacional y regional los procesos de implementación de medidas/iniciativas estructurales y no estructurales que los municipios consideren después de esta evaluación. Además, es esencial comprender que existen diferentes enfoques para la reducción de los factores de riesgo, algunos como la RRD basada en la comunidad, así como alianzas entre gobiernos locales o entre el sector público y el privado que interviene en el territorio.

Dada la naturaleza multidimensional de la encuesta, puede haber múltiples análisis que se deriven de los resultados obtenidos. Para la muestra de 2017 se decidió verificar si existe una relación entre los indicadores obtenidos y algunas condiciones que se reconocen internacionalmente como factores determinantes o de riesgo agravante. La conclusión es que sí existe una relación directa entre: pobreza por ingresos, desarrollo urbano acelerado, planificación territorial deficiente y recursos financieros escasos destinados a mitigar el riesgo presente con condiciones de alta predisposición al riesgo de desastres. Esta conclusión parcial debe validarse siempre que se conozca el comportamiento de todos los municipios presentes en el país con el objeto de detectar tendencias de manera fehaciente a nivel nacional, macrozonal u otra escala geográfica que se desee analizar.

Finalmente, se espera que este instrumento contribuya a la gestión eficaz del riesgo de desastres a nivel local favoreciendo la atención de condiciones de vulnerabilidad potencial que comprometen el desarrollo sostenible del país. Del mismo modo, invita a todos los actores del Sistema de Protección Civil a conocer los resultados, enfocar las medidas y generar alianzas de colaboración para una reducción sostenida e inclusiva del riesgo de desastres.

\section{AGRADECIMIENTOS}

Este trabajo es una traducción al español del artículo "Identifying the underlying risk factors of local communities in Chile" originalmente publicado por Natalia Silva Bustos y Cristobal Mena Amigo en la edición de 2019 del Global Assessment Report on Disaster Risk Reduction (GAR 2019) de Naciones Unidas.

\section{REFERENCIAS}

Briguglio, L. (2003). Methodological and practical considerations for constructing socio-economic indicators to evaluate disaster risk, IADB/IDEA Program on Indicators for Disaster Risk Management. Manizales: Universidad Nacional de Colombia, Sede Manizales.

Carreño, M.L., Cardona, O.D., \& Barbat, A.H. (2007). A disaster risk management performance index. Natural Hazards, 41(1), 1-20. https://doi.org/10.1007/s11069-0o6-9008-y

Contreras, E. \& Pacheco, J.F. (2007). Evaluación Multicriterio para Programas y Proyectos Públicos. Recuperado de: http://www.sistemaspublicos.cl/wp-content/files_mf/127258066992-ceges-EC.pdf [visitado el 20 de jul. 2019]

DARA. (2013). RRI Índice de Reducción del Riesgo en África Occidental, Cabo Verde - Gambia - Ghana Guinea - Níger - Senegal, Análisis de las Capacidades y Condiciones para la Reducción del Riesgo de Desastres. Madrid: Fundación DARA Internacional

Dilley, M., Chen, R. S., Deichmann, U., Lerner-Lam, A. L., Arnold, M., Agwe, J., ... Yetman, G. (2005). Natural disaster hotspots: A global risk analysis (No. 34423; pp. 1-145). Recuperado de: http:// documents.worldbank.org/curated/en/621711468175150317/Natural-disaster-hotspots-A-global-riskanalysis [visitado el 3 de nov. 2019]

Lavell, A. (2000). Desastres y desarrollo: hacia un entendimiento de las formas de construcción social de un desastre. El caso de Mitch en Centroamérica. En: BID. Revista del desastre al desarrollo sostenible: huracán Mitch en Centroamérica. San José de Costa Rica: BID \& CIDHS. Recuperado de: http://www.desenredando.org/public/articulos/200o/dyd/DyD200o_mar-1-2002.pdf [visitado el 3 de nov. 2019]

O’Keefe, P., Westgate, K., \& Wisner, B. (1976). Taking the naturalness out of natural disasters. Nature, 260, 566-567.

ONEMI. (2014). Política Nacional para la Gestión del Riesgo de Desastres. Santiago de Chile: ONEMI. 
ONEMI. (2015). Plan Estratégico Nacional para la Gestión del Riesgo de Desastres, 2015-2018. Santiago de Chile: ONEMI.

ONEMI. (2017). Metodología para la Identificación y Caracterización de los Factores Subyacentes del Riesgo de Desastres en el Nivel Comunal. Santiago de Chile: ONEMI. Recuperado de: http:// repositoriodigitalonemi.cl/web/handle/2012/1788 [visitado el 3 de nov. 2019]

Scholz, C.H. (2002). The Mechanics of Earthquakes and Faulting. Cambridge, UK: Cambridge University Press. DOI: https://doi.org/10.1017/9781316681473

Tironi, M. (2014). Atmospheres of indagation: Disasters and the politics of excessiveness. Sociological Review, 62( $\left.\mathrm{S}_{1}\right)$, 114-134.

Twigg, J. (2009). Characteristics of a Disaster-Resilient Community. A Guidance Note. (2da ed.). Londres: UK Department for International Development DFID \& Disaster Risk Reduction Interagency Coordination Group.

United Nations International Strategy for Disaster Reduction UNISDR. (2009). 2009 Global Assessment Report on Disaster Risk Reduction: Risk and poverty in a changing climate. Ginebra: UNISDR.

United Nations International Strategy for Disaster Reduction UNISDR. (2015). Resolution No. 69/283 General Assembly of the United Nations on June 3, 2015, approves Sendai Framework for Disaster Risk Reduction. Ginebra: UNISDR.

UNFCCC. (2015). Framework Convention on Climate Change. Paris: UNFCCC.

UNFCCC. (2017). Opportunities and options for integrating climate change adaptation with the Sustainable Development Goals and the Sendai Framework for Disaster Risk Reduction 2015-2030. Bonn: UNFCCC.

United Nations UN. (2015). Transforming our world: the 2030 Agenda for Sustainable Development. New York: UN.

Wisner, B., Blaikie, P., Cannon, T., \& Davis, I. (2004). At risk: Natural hazards, people's vulnerability, and disasters (2nd ed.). London \& New York: Routledge. 